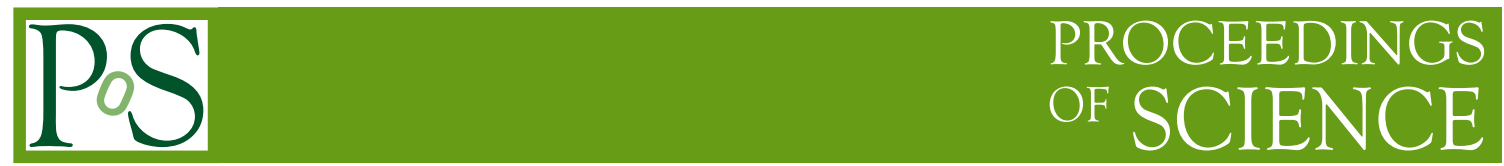

\title{
Multi-channel R-matrix analysis of CNO cycle reactions
}

\author{
E. C. Simpson* \\ University of Surrey \\ E-mail: ph21esesurrey.ac.uk \\ R. E. Azuma
}

University of Notre Dame; University of Toronto

\section{A. Champagne}

University of North Carolina

H. Costantini

University of Notre Dame; INFN Genova

J. Görres

University of Notre Dame

\section{P. J. LeBlanc}

University of Notre Dame

C. Ugalde

University of North Carolina

H.-P. Trautvetter

Institut für Experimentalphysik III, Ruhr-Universität Bochum

M. Wiescher

University of Notre Dame 
The CNO cycle is the main process for hydrogen burning in stars somewhat more massive than the Sun. The reaction cross sections at Gamow energies are typically in the femto to pico-barn range and are consequently very difficult to measure experimentally. The $\mathrm{CNO}$ reaction rates are based on extrapolations of experimental data from higher energies. We are developing a multi-channel R-matrix code (AZURE) to provide a new and more comprehensive tool for fitting experimental data and making extrapolations to lower energies in all reaction and scattering channels.

The ${ }^{14} \mathrm{~N}(\mathrm{p}, \gamma){ }^{15} \mathrm{O}$ reaction is the slowest reaction of the $\mathrm{CNO}$ cycle and thus it determines the energy production rate of $\mathrm{CNO}$ burning. Furthermore, this reaction plays an important role in the determination of Globular Cluster age, since the position of the turnoff point, at which the GC stars escape from the Main Sequence, is powered by the onset of the CNO burning, whose bottleneck is the ${ }^{14} \mathrm{~N}(\mathrm{p}, \gamma){ }^{15} \mathrm{O}$. We have made a reanalysis of the most recent experimental data on the ground state and the $6.18 \mathrm{MeV}$ transitions. The ratio of the cross sections of the ${ }^{15} \mathrm{~N}(\mathrm{p}, \gamma){ }^{16} \mathrm{O}$ and ${ }^{15} \mathrm{~N}(\mathrm{p}, \alpha){ }^{12} \mathrm{C}$ reactions determines how much catalytic material passes to higher CNO cycles and has an effect on the production of heavier elements, particularly ${ }^{16} \mathrm{O}$ and ${ }^{17} \mathrm{O}$. Simultaneous analysis of both reactions for all channels suggests that the ratio $\sigma_{\gamma} / \sigma_{\alpha}$ is smaller than previously reported.

International Symposium on Nuclear Astrophysics - Nuclei in the Cosmos - IX

25-30 June 2006

CERN

*Speaker. 

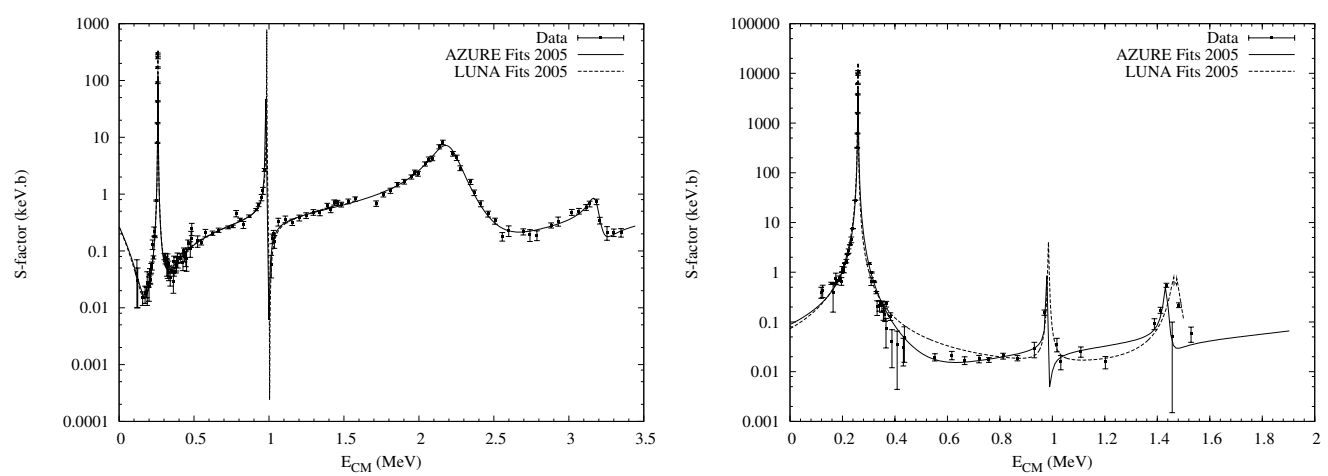

Figure 1: Sample fits for the ground state and 6.18 MeV state radiative capture transitions. The astrophysical $\mathrm{S}$-factor in the ground state transition is determined by the interference between the subthreshold state, the $2.187 \mathrm{MeV} 3 / 2^{+}$state and the contribution from the $3 / 2^{+}$background pole used to simulate direct capture. For the transition to the $6.18 \mathrm{MeV}$ state, the low energy S-factor is principally determined by the interference between the $0.259 \mathrm{MeV} 1 / 2^{+}$resonance and the $1 / 2^{+}$background pole.

\section{1. ${ }^{14} \mathbf{N}(\mathbf{p}, \gamma){ }^{15} \mathrm{O}$ and ${ }^{14} \mathbf{N}(\mathbf{p}, \mathbf{p}){ }^{14} \mathrm{~N}$ reactions}

The ${ }^{14} \mathrm{~N}(\mathrm{p}, \gamma){ }^{15} \mathrm{O}$ reaction is the slowest reaction in the first CNO cycle and as such determines the overall rate of energy production. It has been measured over a wide range of energies (see Ref. [1]), with more recent measurements focusing on the low energy region (Refs. [2, 3, 4]).

The radiative capture transitions to the ground and $6.18 \mathrm{MeV}$ states were simultaneously analysed using the AZURE multichannel R-matrix code (see Ref. [5]). For the ground state transition, a $1 / 2^{+}$resonance was included at $E_{C M}=0.259 \mathrm{MeV}$ and $3 / 2^{+}$resonances at $-0.504,0.985$ and 2.187 $\mathrm{MeV}$. Background poles were included at $10 \mathrm{MeV}$ for $\mathrm{J}^{\pi}=1 / 2^{+}$and $3 / 2^{+}$. Contributions from all allowed spins and angular momenta were considered and all reduced-width amplitudes were free to vary. Sample fits can be seen in Fig. 1.

These preliminary results are in good agreement with those published by the LUNA collaboration (Ref. [2, 4]). Further developments of the AZURE code to include direct capture contributions will allow improved analysis of the $\gamma$-ray transitions presented here, as well as the transition to the weakly bound $6.79 \mathrm{MeV}$ state which has the largest contribution to the astrophysical S-factor. Total $(\mathrm{p}, \gamma)$ cross section measurements will also be included in this analysis.

It had been suggested that an analysis of the ${ }^{14} \mathrm{~N}(\mathrm{p}, \mathrm{p}){ }^{14} \mathrm{~N}$ reaction might further constrain the radiative capture channels. An initial analysis of this reaction was made independently of the radiative capture channels, using the data of Ref. [6]. Sample fits for two of the eleven angles available are shown in Fig. 2.

This preliminary elastic scattering analysis has indicated that additional constraint may be placed on the ground state transition. In particular, the reduced-width amplitudes of the broad $3 / 2^{+}$ state at $2.187 \mathrm{MeV}$ should be constrained, helping distinguish the $l=0$ and $l=2$ contributions. It is this state that, along with the subthreshold state and direct capture components, determines the low energy S-factor in the ground state transition. As the ground state is the least well determined transition, further analysis could prove fruitful. There may also be some constraint placed on the contribution from the subthreshold state. 

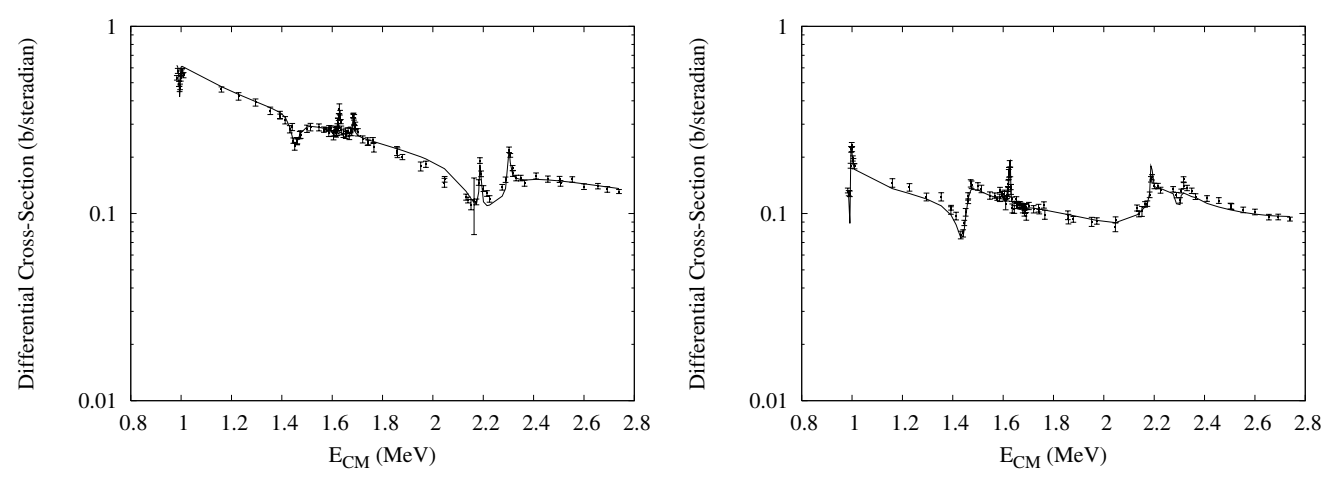

Figure 2: Sample of elastic scattering fits for $\theta=70^{\circ}$ and $\theta=153^{\circ}$.

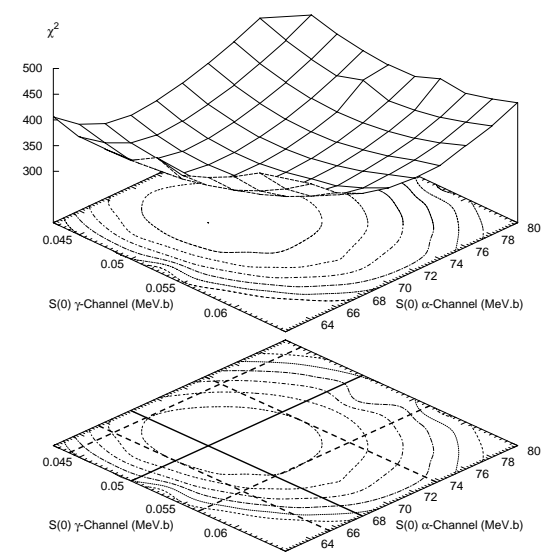

Figure 3: Total $\chi^{2}$ as a function of $\mathrm{S}(0)$ in $\alpha_{0}$ - and $\gamma_{0}$-channels. Contours are placed at $5 \%$ intervals in $\chi^{2}$, with the error arbitrarily taken at $+5 \%$ in the total $\chi^{2}$.

\section{2. ${ }^{15} \mathbf{N}(\mathbf{p}, \gamma){ }^{16} \mathbf{O}$ and ${ }^{15} \mathbf{N}(\mathbf{p}, \alpha){ }^{12} \mathbf{C}$ reactions}

The ${ }^{15} \mathrm{~N}(\mathrm{p}, \gamma){ }^{16} \mathrm{O}$ and ${ }^{15} \mathrm{~N}(\mathrm{p}, \alpha){ }^{12} \mathrm{C}$ reactions are the branching point between the first and second CNO cycles. The data from Refs. [7, 8, 9, 10,11] was reanalysed using a multichannel R-matrix approach. In both the $\alpha_{0}$ - and $\gamma_{0}$-channels the low energy $\mathrm{S}$-factor is determined by the interference between the $1^{-}$resonances at 0.312 and $0.96 \mathrm{MeV}$. Also included were the $1^{+}$and $3^{-}$ resonances at 1.53 and $1.13 \mathrm{MeV}$ respectively, and $1^{-}$and $3^{-}$background poles at $10 \mathrm{MeV}$. All allowed spin and angular momentum channels were considered and all reduced-width amplitudes were free to vary.

Error estimates for the astrophysical S-factors were made by fixing the low energy S-factor, fitting and recording the fitted $\chi^{2}$. The minimum $\chi^{2}$ gives the physical $\mathrm{S}(0)$ and the rate of deviation from the minimum gives an estimate of the error (see Fig. 3). The resulting astrophysical S-factor estimates are $\mathrm{S}_{\gamma_{0}}(0)=50_{-6}^{+7} \mathrm{keV} . \mathrm{b}$ and $\mathrm{S}_{\alpha_{0}}(0)=68.0_{-2.5}^{+5.5} \mathrm{MeV} . \mathrm{b}$. The $\alpha_{0}$-channel result is in good agreement with previous estimates, but the $\gamma_{0}$-channel result is lower than previous estimates of $64 \pm 6 \mathrm{keV} . \mathrm{b}$ (see Ref. [9]). This may be in part due to the background pole used to simulate direct capture. Improvements to the AZURE code to incorporate direct capture are being made and once complete, the result will be checked. 


\section{References}

[1] U. Schröder et. al., Stellar reaction rate of ${ }^{14} N(p, \gamma){ }^{15} O$ and hydrogen burning in massive stars. Nuclear Physics A, 467:240, 1987.

[2] A. Formicola et. al., Astrophysical S-factor of The ${ }^{14} N(p, \gamma)^{15}$ O. Physics Letters B, 591:61, 2004.

[3] R. C. Runkle et. al., Direct Measurement of the The ${ }^{14} N(p, \gamma){ }^{15}$ O S-Factor. Physical Review Letters, 94:082503, 2005.

[4] G. Imbriani et. al., S-factor of The ${ }^{14} N(p, \gamma)^{15} O$ at astrophysical energies. European Physical Journal A - Hadrons and Nuclei, 25:455-466, 2005.

[5] A. M. Lane and R. G. Thomas, R-matrix Theory of Nuclear Reactions, Reviews of Modern Physics, 20:257, 1958.

[6] A. J. Ferguson et. al., Elastic Scattering of Protons by Nitrogen. Atomic Energy of Canada Report PD-261, 1956.

[7] A. Schardt, W. Fowler, and C. C. Lauritsen, The disintegration of ${ }^{15} \mathrm{~N}$ by protons. Physical Review, $86: 527,1952$.

[8] F. Brochard et. al., Étude des Désexcitations Électromagnétiques des Niveaux 1- Situes A 12,44 et 13,09 MeV dans le Noyau ${ }^{16}$ O. Le Journal de Physique, 34:363-367, 1973.

[9] C. Rolfs and W. S. Rodney, Proton Capture by ${ }^{15} \mathrm{~N}$ at stellar Energies. Nuclear Physics A, 235:450, 1974.

[10] J. L. Zyskind and P. D. Parker, Remeasurement of the low-energy cross section for the ${ }^{15} N(p, \alpha)^{12} C$ reaction. Nuclear Physics A, 320:404, 1979.

[11] A. Redder et. al., The ${ }^{15} N(p, \alpha)^{12}$ C Reaction at Stellar Energies. Zeitschrift für Physik A, 305:325, 1982. 